\title{
Solubility and reactivity of HNCO in water: insights into HNCO's fate in the atmosphere
}

\author{
N. Borduas, B. Place, G. R. Wentworth, J. P. D. Abbatt, and J. G. Murphy \\ Department of Chemistry, University of Toronto, Toronto, Ontario, Canada \\ Correspondence to: J. G. Murphy (jmurphy@chem.utoronto.ca)
}

Received: 24 July 2015 - Published in Atmos. Chem. Phys. Discuss.: 7 September 2015

Revised: 2 December 2015 - Accepted: 7 December 2015 - Published: 21 January 2016

\begin{abstract}
A growing number of ambient measurements of isocyanic acid (HNCO) are being made, yet little is known about its fate in the atmosphere. To better understand HNCO's loss processes and particularly its atmospheric partitioning behaviour, we measure its effective Henry's Law coefficient $K_{\mathrm{H}}^{\text {eff }}$ with a bubbler experiment using chemical ionization mass spectrometry as the gas phase analytical technique. By conducting experiments at different $\mathrm{pH}$ values and temperature, a Henry's Law coefficient $K_{\mathrm{H}}$ of $26 \pm 2 \mathrm{M} \mathrm{atm}^{-1}$ is obtained, with an enthalpy of dissolution of $-34 \pm 2 \mathrm{~kJ} \mathrm{~mol}^{-1}$, which translates to a $K_{\mathrm{H}}^{\text {eff }}$ of $31 \mathrm{M} \mathrm{atm}^{-1}$ at $298 \mathrm{~K}$ and at $\mathrm{pH}$ 3. Our approach also allows for the determination of HNCO's acid dissociation constant, which we determine to be $K_{\mathrm{a}}=2.1 \pm 0.2 \times 10^{-4} \mathrm{M}$ at 298 K. Furthermore, by using ion chromatography to analyze aqueous solution composition, we revisit the hydrolysis kinetics of $\mathrm{HNCO}$ at different $\mathrm{pH}$ and temperature conditions. Three $\mathrm{pH}$-dependent hydrolysis mechanisms are in play and we determine the Arrhenius expressions for each rate to be $k_{1}=(4.4 \pm 0.2) \times 10^{7} \exp (-6000 \pm 240 / T) \mathrm{M} \mathrm{s}^{-1}$, $k_{2}=(8.9 \pm 0.9) \times 10^{6} \exp (-6770 \pm 450 / T) \mathrm{s}^{-1} \quad$ and $k_{3}=(7.2 \pm 1.5) \times 10^{8} \exp (-10900 \pm 1400 / T) \mathrm{s}^{-1}$, where $k_{1}$ is for $\mathrm{HNCO}+\mathrm{H}^{+}+\mathrm{H}_{2} \mathrm{O} \rightarrow \mathrm{NH}_{4}^{+}+\mathrm{CO}_{2}, k_{2}$ is for $\mathrm{HNCO}+\mathrm{H}_{2} \mathrm{O} \rightarrow \mathrm{NH}_{3}+\mathrm{CO}_{2}$ and $k_{3}$ is for $\mathrm{NCO}^{-}+2$ $\mathrm{H}_{2} \mathrm{O} \rightarrow \mathrm{NH}_{3}+\mathrm{HCO}_{3}^{-}$. HNCO's lifetime against hydrolysis is therefore estimated to be 10 days to 28 years at $\mathrm{pH}$ values, liquid water contents, and temperatures relevant to tropospheric clouds, years in oceans and months in human blood. In all, a better parameterized Henry's Law coefficient and hydrolysis rates of $\mathrm{HNCO}$ allow for more accurate predictions of its concentration in the atmosphere and consequently help define exposure of this toxic molecule.
\end{abstract}

\section{Introduction}

Until recently, the interest in studying $\mathrm{HNCO}$ was from a fundamental science perspective with research conducted on its structure, preparation and physical properties (Belson and Strachan, 1982) and on its theoretical rovibrational spectra (Mladenović and Lewerenz, 2008). Both theoretical and experimental data indicate that $\mathrm{HNCO}$ is the most stable $\mathrm{CHNO}$ isomer with a near-linear $\pi$-bond system (Hocking et al., 1975; Jones et al., 1950; Poppinger et al., 1977). Roberts et al. (2010) reported detection of HNCO using negative ion proton transfer chemical ionization mass spectrometry (CIMS) from laboratory biomass burning and later determined its emission factor to be $0.25-1.20 \mathrm{mmol}$ per mol of CO for different types of biomass fuels (Veres et al., 2010). Shortly afterwards, the same authors reported the first ambient atmospheric measurements of HNCO in Pasadena, California, reaching $120 \mathrm{pptv}$ and raising concerns of $\mathrm{HNCO}$ exposure due to its toxicity (Roberts et al., 2011). Indeed, HNCO has been observed to cause protein carbamylation leading to cardiovascular disease, rheumatoid arthritis and cataracts (Beswick and Harding, 1984; Lee and Manning, 1973; Mydel et al., 2010; Wang et al., 2007).

Since Roberts et al.'s initial measurements, ambient $\mathrm{HNCO}$ has also been measured in Boulder and in Fort Collins, Colorado, (Roberts et al., 2014), in Toronto, Ontario (Wentzell et al., 2013) and in Calgary, Alberta (WoodwardMassey et al., 2014). HNCO has also been detected simultaneously in the gas phase and in cloud water in La Jolla, California (Zhao et al., 2014). From these studies, typical urban concentrations range from below detection limits to approximately $100 \mathrm{pptv}$, whereas concentrations as high as $1.2 \mathrm{ppbv}$, enough to be of health concern, have been measured in air 
masses impacted by biomass burning in Boulder, Colorado (Roberts et al., 2011, 2014; Woodward-Massey et al., 2014).

HNCO has a variety of anthropogenic and biogenic sources to the atmosphere. HNCO has been quantified from diesel engine exhaust (Kroecher et al., 2005; Wentzell et al., 2013) and light-duty vehicles (Brady et al., 2014) as well as from biogenic sources such as biomass burning (Roberts et al., 2010, 2011, 2014; Veres et al., 2010). There also exist secondary sources of HNCO to the atmosphere, including the gas phase oxidation of amines and amides by $\mathrm{OH}$ radicals producing $\mathrm{HNCO}$ via $\mathrm{H}$-abstraction mechanisms (Barnes et al., 2010; Borduas et al., 2013, 2015). Evidence of secondary sources of HNCO has also been demonstrated in the field, with peak HNCO concentrations occurring during daytime (Roberts et al., 2011, 2014; Zhao et al., 2014).

The sinks of HNCO however remain poorly constrained. $\mathrm{HNCO}$ has a lifetime of decades towards $\mathrm{OH}$ radicals in the atmosphere as estimated by extrapolating high temperature rate coefficients to atmospheric temperatures (Tsang, 1992; Mertens et al., 1992; Tully et al., 1989). It is also not expected to photolyze in the actinic region since its first UV absorption band is observed below $280 \mathrm{~nm}$ wavelengths (Brownsword et al., 1996; Dixon and Kirby, 1968; Rabalais et al., 1969). Nonetheless, HNCO has served as a benchmark system in understanding photodissociation decomposition pathways such as direct and indirect dissociation processes and remains an area of active research (Yu et al., 2013 and references therein). HNCO is most likely removed from the atmosphere by wet and/or dry deposition. HNCO's gas-to-liquid partitioning is therefore an important thermodynamic property that can be used to predict its atmospheric fate. Specifically, the Henry's Law coefficient $K_{\mathrm{H}}$ for the solubility of HNCO represents the equilibrium ratio between its gas phase and aqueous phase concentrations at infinite dilutions according to Eq. (1) (Sander, 2015, 1999). The Henry's Law coefficient for HNCO has only recently been measured by Roberts and coworkers but their experimental set up was limited to a single pH measurement (Roberts et al., 2011). As HNCO is a weak acid with a $\mathrm{pK}_{\mathrm{a}}$ of 3.7 , its Henry's Law coefficient is expected to have a large $\mathrm{pH}$ dependence as described in Eq. (2). Furthermore, the enthalpy of dissolution for HNCO is currently unknown. In lieu of measurements, modelling studies on HNCO have used formic acid's enthalpy of dissolution to model the temperature dependence of HNCO's Henry's Law coefficient (Barth et al., 2013; Young et al., 2012). In our present study, we measure the effective Henry's Law coefficient of $\mathrm{HNCO}$ at a range of $\mathrm{pH}$ and temperatures to determine its enthalpy of dissolution for the first time.

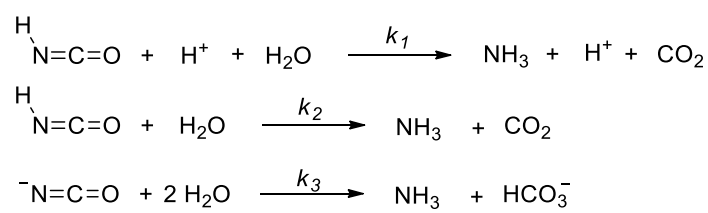

Scheme 1. The three mechanisms involved in HNCO's hydrolysis.

$K_{\mathrm{H}}=C_{\mathrm{HNCO}} / p_{\mathrm{HNCO}}$
$K_{\mathrm{H}}^{\mathrm{eff}}=K_{\mathrm{H}}\left(1+\frac{K_{\mathrm{a}}}{\left[\mathrm{H}^{+}\right]}\right)$.

HNCO reacts irreversibly with water in the aqueous phase, an unusual property for an atmospheric molecule. Once HNCO partitions to the aqueous phase, three mechanisms for its hydrolysis are possible. The first (Reaction R1) is acidcatalyzed and is therefore termolecular, whereas the second (Reaction R2) and third (Reaction R3) are bimolecular reactions involving either the protonated or deprotonated form of HNCO (Scheme 1) (Amell, 1956; Belson and Strachan, 1982; Jensen, 1958). In 1958, Jensen determined the hydrolysis rate of the three mechanisms through addition of $\mathrm{AgNO}_{3}$ to buffered solutions at different time points to precipitate unreacted isocyanate as $\mathrm{AgNCO}$, followed by back titration of excess $\mathrm{AgNO}_{3}$ with $\mathrm{NH}_{4} \mathrm{SCN}$. Considering the importance of these mechanisms in evaluating the fate of $\mathrm{HNCO}$ in the atmosphere, we follow up on the study by Jensen with our own experiments using ion chromatography to determine the $\mathrm{pH}$ and temperature dependencies of the overall rate of hydrolysis of HNCO. Quantitative knowledge of the ability of HNCO to partition to the aqueous phase and its subsequent reactions with water allows for an accurate understanding of the chemical fate of HNCO in the atmosphere (Fig. 1). In this study, we therefore provide laboratory measurements of HNCO's Henry's Law coefficient and enthalpy of dissolution as well as its three rates of hydrolysis and their respective activation energies.

\section{Experimental methods}

\subsection{Henry's Law coefficient experiments}

To measure the effective Henry's Law coefficient $K_{\mathrm{H}}^{\text {eff }}$ of HNCO, we use a bubbler column experimental set up and detect HNCO through chemical ionization mass spectrometry.

\subsubsection{Acetate reagent ion CIMS}

The quadrupole chemical ionization mass spectrometer (CIMS) was built in house and is described in detail elsewhere (Escorcia et al., 2010). We opted to use acetate as 


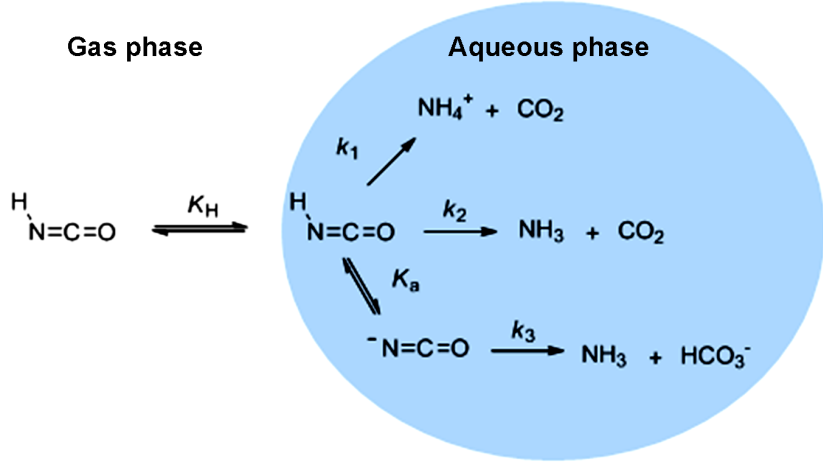

Figure 1. The fate of HNCO in the atmosphere includes its partitioning between the gas and aqueous phases and its hydrolysis through three different mechanisms governed by $k_{1}, k_{2}$, and $k_{3}$.

the reagent ion which has been shown to be sensitive for the detection of acids (Roberts et al., 2010; Veres et al., 2008). For this experimental set up, the reagent ion was generated by flowing $20 \mathrm{sccm}$ of nitrogen over a glass tube containing acetic anhydride (from Sigma-Aldrich and used as is) and maintained at $30^{\circ} \mathrm{C}$. This flow was subsequently mixed with a nitrogen dilution flow of $2 \mathrm{~L} \mathrm{~min}^{-1}$ and passed through a polonium-210 radioactive source to generate acetate ions. All flows were controlled using mass flow controllers. The data acquisition was done under selected ion mode where 10 $\mathrm{m} / \mathrm{z}$ ratios were monitored with dwell times of $0.2 \mathrm{~s}$ each and each duty cycle was $4 \mathrm{~s}$. Ions measured included $\mathrm{m} / z 59$ $\left(\mathrm{AcO}^{-}\right), m / z 119\left((\mathrm{AcOH}) \mathrm{AcO}^{-}\right), m / z 51$ (black counts), $m / z 35\left(\mathrm{Cl}^{-}\right), m / z 42\left(\mathrm{NCO}^{-}\right), m / z 45\left(\mathrm{HCOO}^{-}\right), m / z 46$ $\left(\mathrm{ONO}^{-}\right), m / z 96, m / z 102$ and $m / z 113$. The raw signals are then normalized to $m / z 59$ and reported as normalized counts per second (ncps). For the exception of $m / z 42$, none of the ions were observed to change during the experiments. The inlet flow of the CIMS is governed by a pin hole at $0.5 \mathrm{~L} \mathrm{~min}^{-1}$, and a $\mathrm{N}_{2}$ dilution flow of $0.4 \mathrm{~L} \mathrm{~min}^{-1}$ into the inlet was used to avoid depletion of the acetate reagent ion by high $\mathrm{HNCO}$ concentrations. Previous work suggests there is no significant role of water vapour in HNCO's detection by acetate CIMS (Roberts et al., 2010). With the CIMS's inlet dilution, the RH within the ion molecule region was $<20 \%$.

\subsubsection{Experimental set-up for measurement of $K_{H}$}

To obtain the Henry's Law coefficient, $K_{\mathrm{H}}$, we monitored the decrease in gas phase HNCO exiting a buffered aqueous solution for a range of volume flow rates. A bubbler column experimental set up is used with online gas phase detection. This method is employed to measure HNCO's partitioning and take into account the concurrent hydrolysis of $\mathrm{HNCO}$ in the buffer solution at high time resolution. Our experimental setup is based on previous work (Kames and Schurath, 1995; Roberts, 2005; Roberts et al., 2011) and our apparatus is comprised of one fritted bubbler with an approx- imate volume of $70 \mathrm{~mL}$ which contained $15 \mathrm{~mL}$ of a citric acid/ $\mathrm{Na}_{2} \mathrm{HPO}_{4}$ buffer at varying $\mathrm{pH}$. The $15 \mathrm{~mL}$ volume was chosen to reduce HNCO equilibration times and to simultaneously ensure that the bubbler's frit was submerged. Experiments performed in $30 \mathrm{~mL}$ of buffer yielded identical results. The water lost to the gas phase during the experiments $(<1 \mathrm{~h})$ was at most $5 \%$ of the original buffer volume and so no corrections to the latter were required. The bubbler was held in a temperature-controlled bath of approximately $1: 1$ mixture of deionized water and ethylene glycol. Upstream of the bubbler, where the RH was measured to be $\sim 50 \%$, was a valve and a tee connection where the dry HNCO flow could be connected and disconnected during the experiments. Downstream of the bubbler was another tee which connected to both the exhaust and the acetate reagent ion CIMS. Conveniently, the absolute concentration of gas-phase HNCO is not required in this approach since it relies on the decay of the signal, $[\mathrm{HNCO}]_{t} /[\mathrm{HNCO}]_{0}$ and not on the absolute gas phase and aqueous phase concentrations.

$\mathrm{HNCO}$ was produced using a permeation source which sublimes solid cyanuric acid at $250^{\circ} \mathrm{C}$ in a flow of dry nitrogen and is described in detail elsewhere (Borduas et al., 2015). This source is based on HNCO sublimation techniques and on a similar source previously developed by Roberts et al. (Belson and Strachan, 1982; Roberts et al., 2010). The buffer solutions were made with solid citric acid, disodium phosphate and deionized water with citric acid concentrations ranging from 0.02 to $0.0035 \mathrm{M}$ to access a $\mathrm{pH}$ range of 2.5-4.0.

Each experiment began with gaseous HNCO flowing through a fresh buffer solution until a reasonably stable signal ( $>0.01 \mathrm{ncps}$ ) was obtained by the CIMS (background counts $\left.\sim 5 \times 10^{-4} \mathrm{ncps}\right)$. The solution did not need to reach equilibrium for the experiment to proceed and so lower temperatures and higher $\mathrm{pHs}$ (when the equilibration time is longest and may reach over $4-5 \mathrm{~h}$ ) were feasible. Once a normalized signal (i.e. relative to the reagent ion signal) of at least 0.025 for HNCO was obtained, the flow of HNCO through the bubbler was turned off, and only pure nitrogen continued to flow through. The HNCO signal then decayed exponentially as a function of time due to partitioning as well as hydrolysis. This decay was monitored until it had decreased to less than one quarter of the original signal. This method also has the advantage of extracting an effective Henry's Law coefficient without needing to monitor the aqueous phase $\mathrm{HNCO}$ concentration.

\subsection{Hydrolysis rate experiments}

HNCO in the aqueous phase was measured using ion chromatography at different $\mathrm{pH}$ and temperatures to determine its rates of hydrolysis. 


\subsubsection{Ion chromatography}

The measurements for the hydrolysis of HNCO were made using a Dionex IC-2000 Ion Chromatography (IC) System. An IonPac (AS19) anion column consisting of a quaternary ammonium ion stationary phase with diameter and length dimensions of 4 and $25 \mathrm{~mm}$ respectively was employed. Sample runs used a concentration gradient of the eluent $\mathrm{KOH}$ ranging from 2 to $20 \mathrm{mM}$. An optimized elution program was written for each $\mathrm{pH}$ range measured (between 25$60 \mathrm{~min}$ for each injection). Samples were injected using a Dionex (AS40) automated sampler into a $25 \mu \mathrm{L}$ loop for preinjection. The use of a loop rather than a concentrator was important and ensured that the total $\mathrm{HNCO} / \mathrm{NCO}^{-}$concentrations were being measured. The IC was calibrated using matrix-matched standards of known $\mathrm{HNCO} / \mathrm{NCO}^{-}$concentrations prepared from serial dilutions of KOCN (SigmaAldrich, $96 \%$ purity).

\subsubsection{Hydrolysis kinetics experiments}

The kinetics of the hydrolysis reactions in the $\mathrm{pH}$ range of 12 are very fast; complete decays occurred in a matter of minutes. The decay of HNCO at these low $\mathrm{pH}$ values is therefore too quick for the $25 \mathrm{~min}$ IC method to capture. To circumvent this issue we used a quenching method. Specifically, we prepared an aqueous solution of $50 \mathrm{~mL}$ of sulphuric acid at the desired pH. $5 \mathrm{~mL}$ of this acidic solution was subsequently added to a $0.02 \mathrm{M}$ solution of $\mathrm{KOCN}$ in eight different falcon tubes to initiate the rapid hydrolysis reaction. Each reaction was then quenched at different times by a $0.1 \mathrm{M}$ aqueous solution of $\mathrm{KOH}$. Increasing the $\mathrm{pH}$ to more than 10 slowed the hydrolysis kinetics by orders of magnitude and allowed for subsequent IC measurements. Replacing sulphuric acid by nitric acid and/or $\mathrm{KOH}$ by $\mathrm{NaOH}$ yielded identical hydrolysis rates and ensured the results were reproducible with different acids and bases.

Buffer solutions in the $\mathrm{pH}$ range of 3-5 were prepared by using appropriate molar ratios of citric acid and disodium phosphate whereas buffer solutions in the $\mathrm{pH}$ range of 9-10 used sodium carbonate and sodium bicarbonate. All buffer concentrations were $<0.002 \mathrm{M}$, and we assume that the ionic strength of these solutions had minimal impact on the solubility of HNCO. For the room temperature set of kinetic experiments, the experiment was initiated by the addition of $0.1 \mathrm{~g}$ of KOCN to $50 \mathrm{~mL}$ of the desired buffer solution. The solution was further diluted by a factor of 500 and then split into eight samples for analysis at succeeding intervals on the anion IC.

Hydrolysis reactions were run at different temperatures to assess the activation energies of each of the three hydrolysis mechanisms. Room temperature reactions were conducted inside the IC autosampler AS40 (with a cover) and monitored by a temperature button (iButtons, Maxim Integrated, San Jose, CA with $0.5^{\circ} \mathrm{C}$ resolution). Colder temperature re-
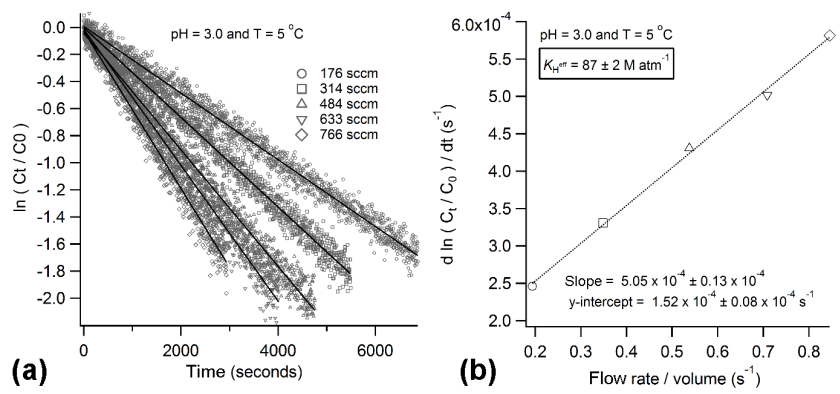

Figure 2. (a) The concentration decay curves as a function of time according to Eq. (4) for each flow rate shown; (b) the slopes of each fit in (a) plotted as a function of the ratio of the flow rate to the volume. The symbols in both figures represent the same flow rate shown.

actions were done in a water ice bath and monitored by a thermometer. Finally, warmer temperature reactions for high $\mathrm{pH}$ samples were run in a temperature-controlled water bath. These reactions took days to weeks to reach completion, and so $5 \mathrm{~mL}$ samples from the reaction mixtures were taken out of the water bath and measured on the IC at appropriate time intervals.

\section{Results and discussion}

\subsection{Henry's Law coefficient $K_{H}$}

HNCO's effective Henry's Law solubility coefficient $K_{\mathrm{H}}^{\mathrm{eff}}$ expressed in $\mathrm{M} \mathrm{atm}^{-1}$ was determined based on the exponential decay of gaseous HNCO exiting a bubbler containing a buffered solution. The observed decay of HNCO is caused by its partitioning from the aqueous phase to the gas phase as well as its competing hydrolysis reaction. Equation (3) represents the rate law for the disappearance of $\mathrm{HNCO}$ during the experiment and Eq. (4) is the integrated rate law.

$$
\begin{aligned}
& -\frac{d[\mathrm{HNCO}]}{\mathrm{d} t}=[\mathrm{HNCO}] \frac{\varphi}{K_{\mathrm{H}}^{\mathrm{eff}} \mathrm{VRT}}+[\mathrm{HNCO}] k_{\mathrm{hyd}} \\
& \ln \frac{[\mathrm{HNCO}]_{t}}{[\mathrm{HNCO}]_{0}}=-\left[\frac{\varphi}{K_{\mathrm{H}}^{\mathrm{eff}} \mathrm{VRT}}+k_{\mathrm{hyd}}\right] t
\end{aligned}
$$

where $[\mathrm{HNCO}]_{t}$ is the $\mathrm{HNCO}$ concentration at time $t$, $[\mathrm{HNCO}]_{0}$ is the initial HNCO concentration (at time $t=0$ ), $[\mathrm{HNCO}]_{t} /[\mathrm{HNCO}]_{0}$ is the $\mathrm{HNCO}$ concentration in the gas phase downstream of the bubbler measured by the CIMS, $\phi$ is the volumetric flow rate $\left(\mathrm{cm}^{3} \mathrm{~s}^{-1}\right), K_{\mathrm{H}}^{\text {eff }}$ is the effective Henry's Law coefficient for solubility $\left(\mathrm{mol} \mathrm{L}^{-1} \mathrm{~atm}^{-1}\right), V$ is the liquid volume of the buffer $\left(\mathrm{cm}^{3}\right), R$ is the ideal gas constant $\left(8.21 \times 10^{-2} \mathrm{~L} \mathrm{~atm} \mathrm{~mol}^{-1} \mathrm{~K}^{-1}\right), T$ is the temperature $(\mathrm{K}), k_{\text {hyd }}$ is HNCO's overall rate of hydrolysis $\left(\mathrm{s}^{-1}\right)$ and $t$ is the time (s). To extract the value of $K_{\mathrm{H}}^{\mathrm{eff}}$ from the experimental decay curves, we first plot the natural logarithm of change 

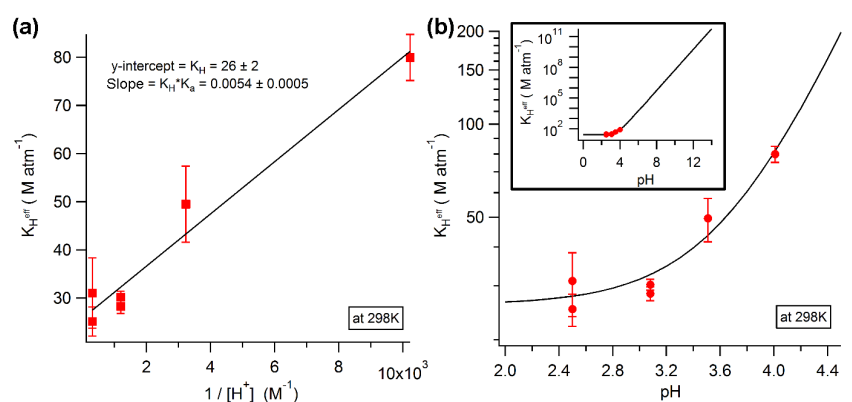

Figure 3. (a) The fit according to Eq. (2) of the experimental $K_{\mathrm{H}}^{\mathrm{eff}}$ values which allows for the determination of $K_{\mathrm{H}}$ and $K_{\mathrm{a}}$ at $298 \mathrm{~K}$. (b) The experimental $K_{\mathrm{H}}^{\mathrm{eff}}$ values as a function of $\mathrm{pH}$ at $298 \mathrm{~K}$. The black line is the modelled dependence of $K_{\mathrm{H}}^{\text {eff }}$ according to Eq. (2) based on the determined value of $K_{\mathrm{H}}$ and a value for $K_{\mathrm{a}}$ of $2.1 \times 10^{-4} \mathrm{M}$. The inset shows the range of $K_{\mathrm{H}}^{\mathrm{eff}}$ across the full range of $\mathrm{pH}$.

in $\mathrm{HNCO}$ concentration versus time for different flow rates ranging from 175 to $800 \mathrm{sccm}$ as shown in Fig. 2a. The slope of each experiment is then plotted as function of the ratio of the flow rate and volume depicted in Fig. $2 \mathrm{~b}$. The slope of Fig. $2 \mathrm{~b}$ leads to a value representing $\left(K_{\mathrm{H}}^{\text {eff }} \mathrm{RT}\right)^{-1}$ and so $K_{\mathrm{H}}^{\text {eff }}$ can be calculated.

These dynamic experiments were repeated with a range of buffer solutions ranging from $\mathrm{pH} 2.5$ to 4.0 to determine the pH-independent Henry's Law coefficient, $K_{\mathrm{H}}$, of HNCO. Experiments at temperatures of 273-298 K were also conducted to determine HNCO's enthalpy of dissolution, $\Delta \mathrm{H}_{\mathrm{diss}}$.

\subsection{1 pH dependence of $K_{\mathrm{H}}^{\text {eff }}$}

The $\mathrm{pH}$ dependence of the effective Henry's Law coefficient $K_{\mathrm{H}}^{\text {eff }}$ of a weak acid like HNCO depends on its $\mathrm{pK}_{\mathrm{a}}$ as well as on the $\mathrm{pH}$ according to Eq. (2). Throughout our experiments, we measure the value of $K_{\mathrm{H}}^{\text {eff }}$ and employ Eq. (2) to plot $K_{\mathrm{H}}^{\text {eff }}$ as a function of the inverse of the proton concentration, $\left[\mathrm{H}^{+}\right]$, and thus to extract HNCO's Henry's Law coefficient for solubility, $K_{\mathrm{H}}$. Figure $3 \mathrm{a}$ depicts this linear relationship and yields a value of $26 \pm 2 \mathrm{M} \mathrm{atm}^{-1}$ for $K_{\mathrm{H}}$. Our $K_{\mathrm{H}}$ value compares well with the only other published value of $21 \mathrm{M} \mathrm{atm}^{-1}$ determined solely at $\mathrm{pH} 3$ (Roberts et al., 2011). Figure $3 \mathrm{~b}$ on the other hand shows experimentally determined $K_{\mathrm{H}}^{\text {eff }}$ at different $\mathrm{pH}$ values and at a constant temperature of $298.0 \pm 0.2 \mathrm{~K}$. Error bars in both Fig. 3a and $\mathrm{b}$ represent the percentage of the standard deviation of the slope as in Fig. 2b. The slope in Fig. 3a also allows us to determine HNCO's acid dissociation constant, $K_{\mathrm{a}}$, which at $298 \mathrm{~K}$ is $2.1 \pm 0.2 \times 10^{-4} \mathrm{M}$. Our $K_{\mathrm{a}}$ value also agrees well with previously reported $K_{\mathrm{a}}$ for $\mathrm{HNCO}$ (Amell, 1956; Belson and Strachan, 1982).

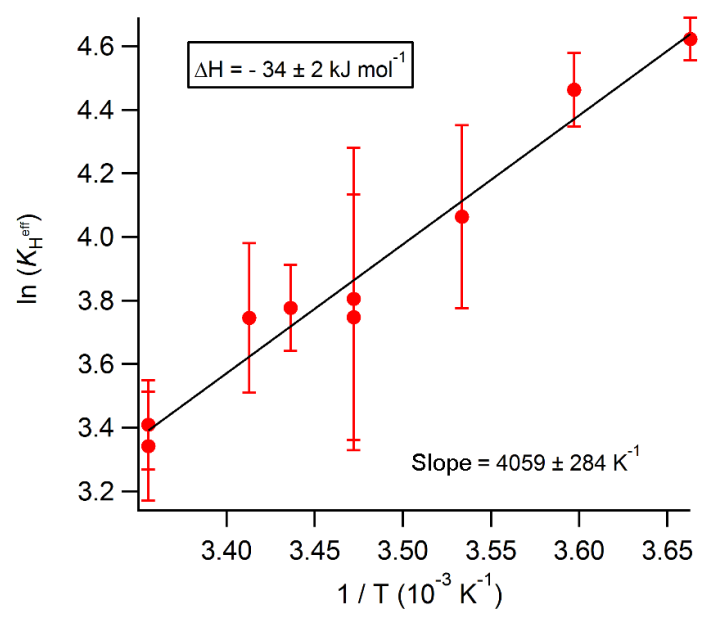

Figure 4. The temperature dependence of experimentally measured $K_{\mathrm{H}}^{\mathrm{eff}}$ at $\mathrm{pH} 3.08$.

\subsubsection{Temperature dependence}

The temperature dependence of HNCO's solubility was established by running experiments at varying temperatures from 273 to $298 \mathrm{~K}$. Since $K_{\mathrm{H}}^{\mathrm{eff}}$ is very sensitive to $\mathrm{pH}$ changes, all experiments were conducted with a buffer solution from the same batch and same volumetric flask within a few days. Plotting the natural logarithm of the effective Henry's Law coefficient as a function of the inverse of temperature yields the ratio of the enthalpy of dissolution, $\Delta \mathrm{H}_{\text {diss }}$, to the gas constant, $R$ (Fig. 4). We report a value of $-34 \pm 2 \mathrm{~kJ} \mathrm{~mol}^{-1}$ for HNCO's enthalpy of dissolution, where the uncertainty stems from the deviation from the slope depicted in Fig. 4. This value compares to similar weak acids like $\mathrm{HONO}\left(-40 \mathrm{~kJ} \mathrm{~mol}^{-1}\right)$ and $\mathrm{HCN}\left(-42 \mathrm{~kJ} \mathrm{~mol}^{-1}\right)$, but differs from the value of formic acid $\left(-47 \mathrm{~kJ} \mathrm{~mol}^{-1}\right)$ which was the value assumed for HNCO in the Young et al., and the Barth et al., modelling studies (Barth et al., 2013; Sander, 2015; Young et al., 2012).

\subsection{Rate of hydrolysis $k_{\text {hyd }}$}

There are three mechanisms by which HNCO can react with water described in Scheme 1 Reaction (R1) to (R3) and depicted in Fig. 1. The disappearance of HNCO in the aqueous phase can therefore be described by the rate law shown as Eq. (5). The $\mathrm{pH}$ dependence of HNCO's hydrolysis manifests itself in the first term of Eq. (5) as the hydrogen ion concentration as well as in the concentration of the dissociated and/or non-dissociated acid in each term.

$$
\begin{aligned}
-\frac{d[\mathrm{HNCO}]_{t}}{\mathrm{~d} t}= & k_{1}[\mathrm{HNCO}]\left[\mathrm{H}^{+}\right]+k_{2}[\mathrm{HNCO}] \\
& +k_{3}\left[\mathrm{NCO}^{-}\right] .
\end{aligned}
$$

To mathematically integrate this rate law, the concentration of HNCO needs to be expressed as the sum of undis- 
sociated $\mathrm{HNCO}$ and of isocyanate ion $\mathrm{NCO}^{-}$in solution, which is denoted in Eq. (6) as [HNCO $]_{\text {tot }}$. HNCO's acid dissociation constant $K_{\mathrm{a}}$ relates the concentration of $\mathrm{HNCO}$ and $\mathrm{NCO}^{-}$as shown in Eq. (6). The $K_{\mathrm{a}}$-dependant expression of Eq. (6) is then substituted into the rate law of Eq. (5), and subsequently integrated. The $K_{\mathrm{a}}$ value of $\mathrm{HNCO}$ has a slight temperature dependence with a heat of dissociation previously measured to be $5.4 \mathrm{~kJ} \mathrm{~mol}^{-1}$, which for the temperature range of 273 to $298 \mathrm{~K}$ represents a $25 \%$ change (Amell, 1956). We therefore use Amell's heat of dissociation value throughout our analysis to account for $K_{\mathrm{a}}$ 's temperature dependence in the van't Hoff equation. Furthermore, Belson et al.'s evaluation of the $K_{\mathrm{a}}$ of HNCO literature recommends $2.0 \times 10^{-4} \mathrm{M}$ at $298 \mathrm{~K}$ (Belson and Strachan, 1982). Finally, our own work on the $\mathrm{pH}$ dependence of Henry's Law coefficient of HNCO, suggests a $K_{\mathrm{a}}$ value of $2.1 \pm 0.2 \times 10^{-4} \mathrm{M}$ at $298 \mathrm{~K}$, consistent with the recommended value (Fig. 3a).

$$
\begin{aligned}
{[\mathrm{HNCO}]=} & {[\mathrm{HNCO}]_{\mathrm{tot}}-\left[\mathrm{NCO}^{-}\right]=\frac{\left[\mathrm{H}^{+}\right]\left[\mathrm{NCO}^{-}\right]}{K_{\mathrm{a}}}=} \\
& \frac{[\mathrm{HNCO}]_{\mathrm{tot}}\left[H^{+}\right]}{K_{\mathrm{a}}+\left[\mathrm{H}^{+}\right]} .
\end{aligned}
$$

By integrating Eq. (5) with the appropriate substitutions, the resulting expression is Eq. (7), where $k_{\text {hyd }}$ represents the observed first-order rate loss of hydrolysis of HNCO and depends on the individual reaction rates $k_{1}, k_{2}$ and $k_{3}$ according to Eq. (8).

$$
\begin{aligned}
& \frac{[\mathrm{HNCO}]_{t}}{[\mathrm{HNCO}]_{0}}=e^{-k_{\mathrm{hyd}} t} \\
& k_{\mathrm{hyd}}=\frac{k_{1}\left[\mathrm{H}^{+}\right]^{2}+k_{2}\left[\mathrm{H}^{+}\right]+k_{3} K_{\mathrm{a}}}{K_{\mathrm{a}}+\left[\mathrm{H}^{+}\right]} .
\end{aligned}
$$

The aim of our hydrolysis experiments is to measure $k_{\text {hyd }}$ at different $\mathrm{pH}$ values to subsequently solve for the values of the individual hydrolysis rate coefficients $k_{1}, k_{2}$ and $k_{3}$. To measure $k_{\text {hyd }}$, we employ ion chromatography (IC) which allows for quantitative measurement of the total isocyanic acid in solution as $\mathrm{NCO}^{-}$using an anion chromatography column. The key to making $[\mathrm{HNCO}]_{\text {tot }}$ measurements was to use a loop injection port for the IC instead of a concentrator column, since the latter retains only ions and would not measure any protonated HNCO in solution. Appropriate buffer solutions were made to conduct experiments over a range of $\mathrm{pH}$ values from 1.7 to 10.4 . The decay of [HNCO] tot was monitored by IC over time and plotting the natural logarithm of the decay as a function of time as in Fig. 5 yields the $k_{\text {hyd }}$ specific to that temperature and $\mathrm{pH}$. Hydrolysis experiments are listed in Table A1 in Appendix A.

\subsubsection{Determining $k_{1}$ and $k_{2}$}

At a pH below 3, the third hydrolysis mechanism (Scheme 1 (Reaction R3)) will contribute minimally to the overall $k_{\text {hyd }}$. Indeed, the third term in Eq. (8), $k_{3} K_{\mathrm{a}} /\left(K_{\mathrm{a}}+\left[\mathrm{H}^{+}\right]\right)$

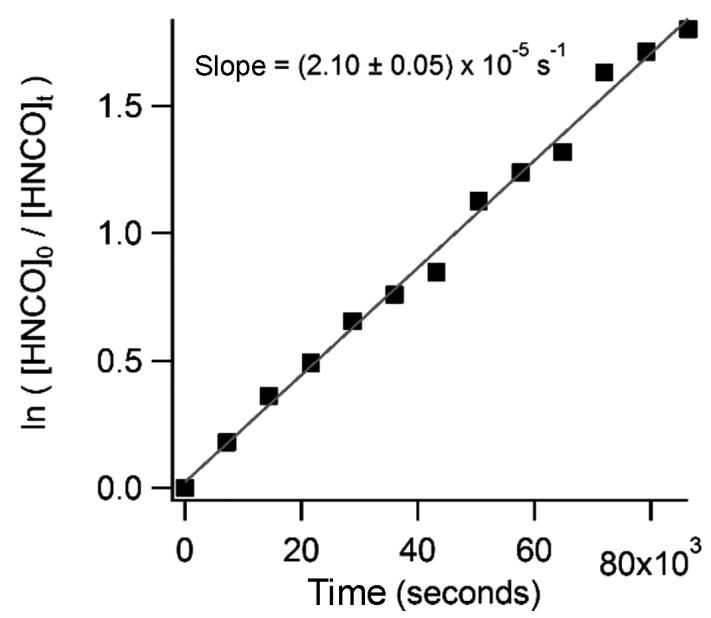

Figure 5. Example of a hydrolysis experiment at $\mathrm{pH} 5.4$ and at $25^{\circ} \mathrm{C}$ where the $[\mathrm{HNCO}]_{\text {tot }}$ is measured by loop injections on the IC.

will become very small because $\left[\mathrm{H}^{+}\right] \gg K_{\mathrm{a}}$. Furthermore, very little of the $\mathrm{HNCO}$ is present as $\mathrm{NCO}^{-}$at low $\mathrm{pH}$. This assumption (which we verify retroactively) simplifies the $k_{\text {hyd }}$ expression to Eq. (9) with only two unknowns, $k_{1}$ and $k_{2}$. We can now solve for $k_{1}$ and $k_{2}$ from two $k_{\text {hyd }}$ values derived from experiments conducted at two different $\mathrm{pH}$ values but at the same temperature. For example, solving for $k_{1}$ and $k_{2}$ at $295 \mathrm{~K}$ using the $k_{\text {hyd }}$ in Table A1, we obtain a value of $(6.73 \pm 0.27) \times 10^{-2} \mathrm{M} \mathrm{s}^{-1}$ for $k_{1}$ and of $(1.04 \pm 0.04) \times 10^{-3} \mathrm{~s}^{-1}$ for $k_{2}$. We do this calculation once per temperature. The uncertainties associated with these measurements come from the slope of decay of aqueous phase HNCO measured by IC.

$k_{\text {hyd } @ \mathrm{pH}<2.7}=\frac{k_{1}\left[\mathrm{H}^{+}\right]^{2}+k_{2}\left[\mathrm{H}^{+}\right]}{K_{\mathrm{a}}+\left[\mathrm{H}^{+}\right]}$.

\subsubsection{Temperature dependence of $k_{1}$ and $k_{2}$}

Hydrolysis experiments of $\mathrm{HNCO}$ at three different temperatures further enables us to solve for the temperature dependence of $k_{1}$ and $k_{2}$. We chose three temperatures relevant to tropospheric air masses: 270, 283 and 295 K. Figure 6 represents the slope of the natural logarithm of the rate coefficient of hydrolysis as a function of the inverse of the temperature which according to the Arrhenius equation shown in Eq. (10) yields the activation energy specific to each hydrolysis mechanism. We obtain activation energies of $50 \pm 2 \mathrm{~kJ} \mathrm{~mol}^{-1}$ and $56 \pm 4 \mathrm{~kJ} \mathrm{~mol}^{-1}$ for $k_{1}$ and $k_{2}$ respectively. Furthermore, the $y$-intercept of these linear plots yields the value of $\ln (A)$ in Eq. (10) and so the A factors of each hydrolysis mechanism can also be obtained, providing Arrhenius expressions of $k_{1}=(4.4 \pm 0.2) \times 10^{7} \exp (-6000 \pm 240 / T) \mathrm{M} \mathrm{s}^{-1}$ and $k_{2}=(8.9 \pm 0.9) \times 10^{6} \exp (-6770 \pm 450 / T) \mathrm{s}^{-1}$. The uncertainties stem from the fit to the data points in Fig. 6 (and their error bars come from the slope of the decay of aqueous 


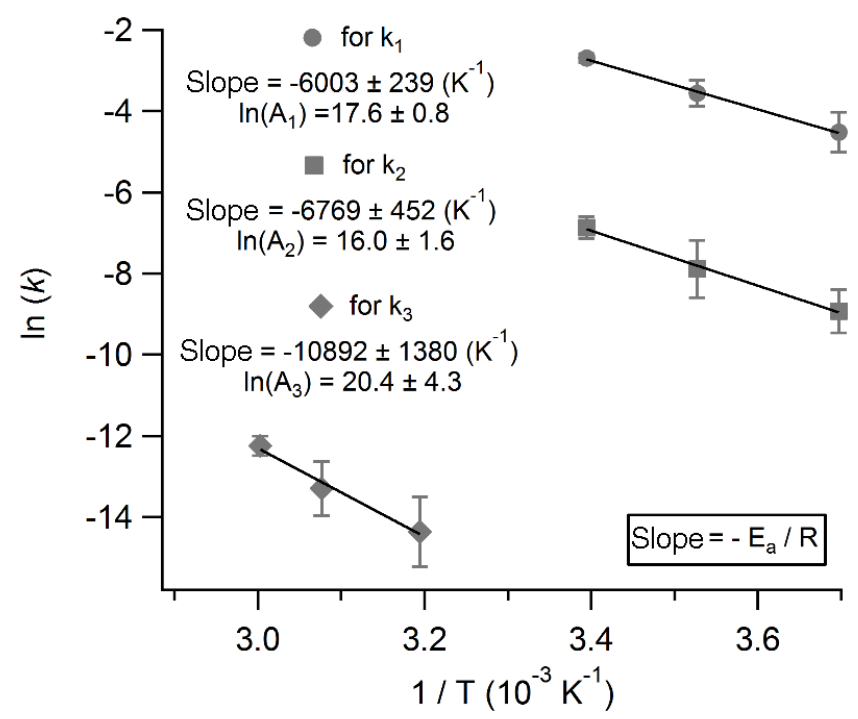

Figure 6. The linear plots of the natural logarithm of each hydrolysis rate coefficient $k_{1}, k_{2}$ and $k_{3}$ as a function of the inverse of temperature to yield the activation energies of each mechanism.

phase HNCO measured by IC).

$k=A e^{-E_{\mathrm{a}} / R T}$

\subsubsection{Determining $k_{3}$ and its temperature dependence}

At high $\mathrm{pH}$ levels, the third hydrolysis mechanism (Scheme 1 (Reaction R3)) will dominate the observed $k_{\text {hyd }}$, however, the first two mechanisms may still have a non-negligible contribution to $k_{\text {hyd }}$ and can therefore not be disregarded. We can solve for $k_{3}$, knowing $k_{1}$ and $k_{2}$ and their respective temperature dependencies, using Eq. (8). The $k_{\text {hyd }}$ values measured at $\mathrm{pH}$ above 9 and at $40^{\circ} \mathrm{C}$ are used (Table A1), and $k_{3}$ is determined for each $\mathrm{pH}$. The average of our three measurements at $40^{\circ} \mathrm{C}$ is $(5.77 \pm 0.35) \times 10^{-7} \mathrm{~s}^{-1}$. The temperature dependence of $k_{3}$ is determined in an analogous way to $k_{1}$ and $k_{2}$ and is also depicted in Fig. 6. We obtain a value of $91 \pm 12 \mathrm{~kJ} \mathrm{~mol}^{-1}$ which translates to an Arrhenius expression of $k_{3}=(7.2 \pm 1.5) \times 10^{8} \exp (-10900 \pm 1400 / T) \mathrm{s}^{-1}$.

Equipped with the values of $k_{1}, k_{2}$ and $k_{3}$ and their temperature dependencies, a map of the expected total hydrolysis rate, $k_{\text {hyd }}$, as a function of temperature and $\mathrm{pH}$ can be generated using Eqs. (8) and (10) and is plotted as Fig. 7. For reference, the colour scale of Fig. 7 also reads in hydrolysis lifetime of HNCO in hours. It is clear that HNCO's lifetime in the aqueous phase has a large temperature and $\mathrm{pH}$ dependence.

\subsubsection{Comparing the rate of hydrolysis $k_{\text {hyd }}$ through different methods}

The individual rate coefficients of the three hydrolysis mechanisms (Scheme 1, Reactions R1 to R3) have only been evaluated one other time in the literature (Jensen 1958). Our IC experimental method differs substantially from Jensen's back titration method, and yet we obtain similar values for $k_{1}, k_{2}$ and $k_{3}$ as well as for their respective activation energies. The values are summarized in Table 1. Again, the colour scale of Fig. 7 is generated from Eq. (8) using our obtained values for $k_{1}, k_{2}$ and $k_{3}$ and for $E_{\mathrm{a} 1}, E_{\mathrm{a} 2}$ and $E_{\mathrm{a} 3}$, and we superimpose all our $k_{\text {hyd }}$ measurements from Table A1 as circles. We further add Jensen's published raw data for comparison (Jensen, 1958) as triangles. The agreement is good and is consistently within the same order of magnitude (Fig. 7).

In addition, our Henry's Law coefficient experiment provides a complimentary way to determine $k_{\text {hyd }}$ at different temperatures and $\mathrm{pH}$ values. Indeed, the intercept of the line which fit the data of $\mathrm{d} \ln \left(C_{t} / C_{0}\right) / \mathrm{d} t$ vs. $\varphi / V$ yields $k_{\text {hyd }}$, representing the value for the loss process in the solution of the bubbler column experiment (an example is given in Fig. 2b). We show these values as squares in Fig. 7. Roberts et al., also determined $k_{\text {hyd }}$ through this method at $\mathrm{pH} 3$ and at $25^{\circ} \mathrm{C}$ and this value is appended to Fig. 7 (Roberts et al., 2011) as a diamond. The agreement is good from all four cases. We can conclude that the lifetime of HNCO against hydrolysis in dilute aqueous solutions spans seconds to years depending on $\mathrm{pH}$ and temperature. The lifetime of HNCO against hydrolysis in cloud water of $\mathrm{pH} 3-6$ will be shorter and range from $10 \mathrm{~h}$ to $\sim 20$ days in the troposphere. On the other hand, HNCO's hydrolysis in ocean waters of $\mathrm{pH} \sim 8.1$ and temperatures below $30^{\circ} \mathrm{C}$ will be very slow, translating to a lifetime of 1-2 years if we assume no other reactive chemistry is taking place. Finally, in the context of exposure, if HNCO is present in human blood at physiological $\mathrm{pH}$ and temperature, its lifetime to hydrolysis will be as high as several months. On the other hand, if HNCO is present in the stomach, which is more acidic, we would expect its lifetime to drop to minutes or hours.

\section{Atmospheric implications}

HNCO is a toxic molecule and can cause cardiovascular and cataract problems through protein carbamylation (Beswick and Harding, 1984; Mydel et al., 2010; Wang et al., 2007). Recently reported ambient measurements of HNCO in North America raise concerns of exposure particularly from biomass burning, diesel and gasoline exhaust and urban environments (Brady et al., 2014; Roberts et al., 2011, 2014; Wentzell et al., 2013; Woodward-Massey et al., 2014; Zhao et al., 2014). With the values for HNCO's Henry's Law coefficient and hydrolysis rates reported here, a better understanding of HNCO's removal rate from the atmosphere 
Table 1. HNCO's Henry's Law coefficient, acid dissociation constant and hydrolysis constants.

\begin{tabular}{llll}
\hline Physical parameter & Value & Energy & Reference \\
\hline Henry's Law coefficient, $K_{\mathrm{H}}$ & $26 \pm 2 \mathrm{M} \mathrm{atm}^{-1}$ & $\Delta \mathrm{H}_{\mathrm{diss}}=-34 \pm 2 \mathrm{~kJ} \mathrm{~mol}^{-1}$ & This work \\
& $21 \mathrm{M} \mathrm{atm}^{-1}$ & - & Roberts et al. (2011) \\
& $2.1 \pm 0.2 \times 10^{-4} \mathrm{M}$ & - & This work \\
Acid dissociation constant, $K_{\mathrm{a}}^{*}$ & $2.0 \times 10^{-4} \mathrm{M}$ & $\Delta \mathrm{H}_{\mathrm{diss}}=-5.4 \mathrm{~kJ} \mathrm{~mol}^{-1}$ & Amell (1956) \\
& $7.6 \pm 0.3 \times 10^{-2} \mathrm{~s}^{-1}$ & $E_{\mathrm{a} 1}=+50 \pm 2 \mathrm{~kJ} \mathrm{~mol}^{-1}$ & This work \\
Hydrolysis rate coefficient, $k_{1}^{*}$ & $1.1 \times 10^{-1} \mathrm{~s}^{-1}$ & $E_{\mathrm{a} 1}=+63 \mathrm{~kJ} \mathrm{~mol}^{-1}$ & Jensen (1958) \\
& $1.4 \pm 0.1 \times 10^{-3} \mathrm{~s}^{-1}$ & $E_{\mathrm{a} 2}=+56 \pm 4 \mathrm{~kJ} \mathrm{~mol}^{-1}$ & This work \\
Hydrolysis rate coefficient, $k_{2}^{*}$ & $1.8 \times 10^{-3} \mathrm{~s}^{-1}$ & $E_{\mathrm{a} 2}=+83 \mathrm{~kJ} \mathrm{~mol}^{-1}$ & Jensen (1958) \\
& $8.1 \pm 1.7 \times 10^{-8} \mathrm{~s}^{-1}$ & $E_{\mathrm{a} 3}=+91 \pm 12 \mathrm{~kJ} \mathrm{~mol}^{-1}$ & This work \\
Hydrolysis rate coefficient, $k_{3}^{*}$ & $1.2 \times 10^{-8} \mathrm{~s}^{-1}$ & $E_{\mathrm{a} 3}=+100 \mathrm{~kJ} \mathrm{~mol}^{-1}$ & Jensen (1958) \\
\hline
\end{tabular}

* at $298 \mathrm{~K}$

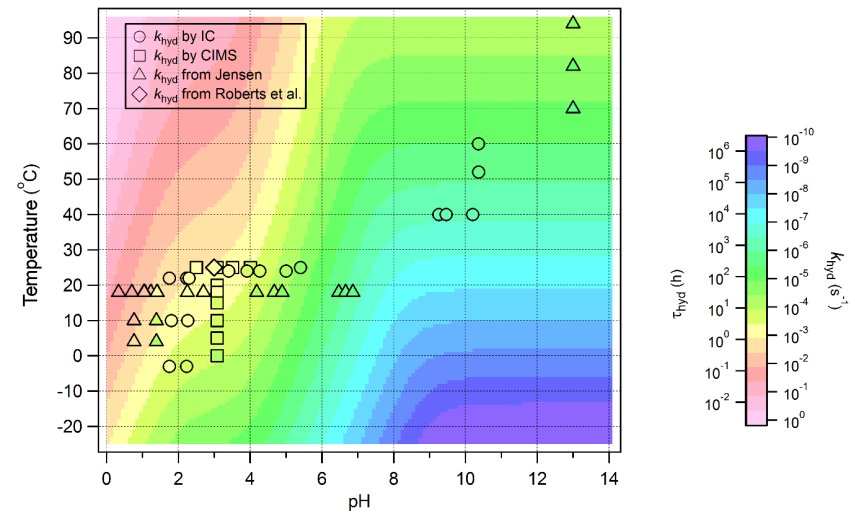

Figure 7. $k_{\text {hyd }}$ as function of temperature and $\mathrm{pH}$ generated from Eq. (8) using our obtained values for $k_{1}, k_{2}$ and $k_{3}$ and for $E_{\mathrm{a} 1}, E_{\mathrm{a} 2}$ and $E_{\mathrm{a} 3}$. All available $k_{\text {hyd }}$ measurements for $\mathrm{HNCO}$ in the literature and from this work are superimposed and colour coded appropriately. As a guide, the colour scale also represents the lifetime in hours for $\mathrm{HNCO}$ in dilute aqueous solutions.

can be determined, and hence HNCO's atmospheric lifetime can be estimated. Note however that our HNCO lifetime estimates do not consider dry deposition and therefore represent a higher limit, particularly since Young et al., found that dry deposition can be significant for HNCO (Young et al., 2012).

Specifically, the lifetime of HNCO in the atmosphere will depend on its partitioning to the aqueous phase $K_{\mathrm{H}}^{\mathrm{eff}}$, the temperature $T$, the $\mathrm{pH}$ and liquid water content (LWC) of the aerosol and/or droplet and finally the hydrolysis of HNCO $k_{\text {hyd }}$ once in solution. We can calculate HNCO's lifetime against hydrolysis based on Eq. (11), where $\tau$ is the lifetime in seconds, $L$ is the fraction of air volume occupied by liquid water (dimensionless) and $R$ is the gas constant. Figure $8 \mathrm{a}$ and $\mathrm{b}$ depict outputs of Eq. (11) with different fixed variables. Figure 8 a holds the LWC to $1 \mathrm{~g} \mathrm{~m}^{-3}$, a value representative of cloud water, highlighting the dependence of HNCO's lifetime on temperature and pH (Ip et al., 2009).

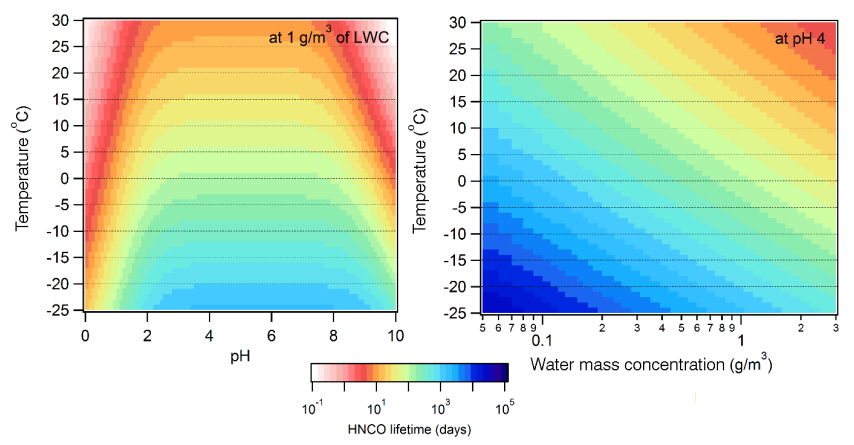

Figure 8. (a) The lifetime of HNCO in days as a function of temperature and $\mathrm{pH}$ at $1 \mathrm{~g} \mathrm{~m}^{-3}$ of LWC and (b) the lifetime of HNCO in days as a function of temperature and $\mathrm{LWC}$ at $\mathrm{pH} 4$.

At atmospherically relevant $\mathrm{pH}$ of 2 to 6 and at temperatures below $30^{\circ} \mathrm{C}, \mathrm{HNCO}$ has a lifetime on the order of 10 days to hundreds of years. Alternatively, Fig. $8 \mathrm{~b}$ holds the $\mathrm{pH}$ at 4 and varies the LWC on the $x$ axis. Water concentrations relevant to wet aerosol $\left(1-100 \mu \mathrm{g} \mathrm{m}^{-3}\right)$ are too small to act as a significant sink for gas phase HNCO. However, Fig. $8 \mathrm{~b}$ highlights the strong dependence of HNCO lifetime on LWC in clouds, again ranging from days to hundreds of years. It therefore appears that if $\mathrm{HNCO}$ is incorporated into cloud water, it is more likely to be rained out or revolatilized than to hydrolyze given typical times in clouds of minutes to hours. There is also the possibility that HNCO has other currently unknown sinks in cloud water that may be competitive with its hydrolysis and further work on HNCO's aqueous phase chemistry with nucleophiles such as amines and alcohols is currently underway in our laboratories. Finally, HNCO will partition readily in oceans at $\mathrm{pH} \sim 8$, but will take years to hydrolyze.

$\tau=1 / K_{\mathrm{H}}^{\mathrm{eff}} R T L k_{\mathrm{hyd}}$. 
Zhao et al. (2014) observed higher concentrations of $\mathrm{HNCO}$ in the cloud water in La Jolla, California than predicted by its Henry's Law coefficient at $298 \mathrm{~K}$ (Zhao et al., 2014). This observation remains puzzling but may point towards sources of $\mathrm{HNCO}$ within cloud water other than simple partitioning chemistry. The Barth et al. (2013) modelling study concluded that fog, low-level stratus clouds or stratocumulus clouds were the most efficient cloud conditions at removing HNCO from the gas phase, particularly in polluted scenarios where the cloud water was more acidic. The authors highlighted the high dependence of HNCO's fate on liquid water $\mathrm{pH}$ and temperature, consistent with our findings (Barth et al., 2013). The Young et al., 2012 study, which modelled global HNCO budgets, assumed the aqueous loss of the weak acid occurred only when the cloud liquid water content was greater than $1 \mathrm{mg} \mathrm{m}^{-3}$. Based on Fig. $8 \mathrm{~b}$, $1 \mathrm{mg} \mathrm{m}^{-3}$ is low for HNCO to significantly partition into the aqueous phase and rather requires water mass concentrations 1000 times greater for HNCO's lifetime to drop to days. The model may have overestimated the ability for LWC to act as a sink for HNCO. HNCO may be a longer lived species than previously thought and exposure of this toxic molecule may pose a threat to regions with HNCO point sources like biomass burning and engine exhaust, as pointed out by Young et al. (2012) and Barth et al. (2013).

\section{Conclusions}

In summary, we provide laboratory measurements of HNCO's important thermochemical properties related to its behavior in water. We measured its Henry's Law coefficient using a bubbler column experiment to be $26 \pm 2 \mathrm{M} \mathrm{atm}^{-1}$ with an enthalpy of dissolution of $-34 \pm 2 \mathrm{~kJ} \mathrm{~mol}^{-1}$. Using ion chromatography, we determined the Arrhenius expression of HNCO's three hydrolysis mechanisms: $k_{1}=(4.4 \pm 0.2) \times 10^{7} \exp (-6000 \pm 240 / T) \mathrm{M} \mathrm{s}^{-1}$, $k_{2}=(8.9 \pm 0.9) \times 10^{6} \exp (-6770 \pm 450 / T) \mathrm{s}^{-1}$ and $k_{3}=$ $(7.2 \pm 1.5) \times 10^{8} \exp (-10900 \pm 1400 / T) \mathrm{s}^{-1}$. These values will provide better constrains on the sinks and thus lifetime of $\mathrm{HNCO}$ in the atmosphere with the aim of minimizing exposure of this toxic molecule. 


\section{Appendix A}

Table A1. Compilation of $k_{\text {hyd }}$ experiments at different $\mathrm{pH}$ and temperatures.

\begin{tabular}{rrl}
\hline $\mathrm{pH}$ & $T(\mathrm{~K})$ & $k_{\text {hyd }}\left(\mathrm{S}^{-1}\right)$ \\
\hline 1.75 & 270 & $(8.30 \pm 0.64) \times 10^{-4}$ \\
1.75 & 295 & $(2.22 \pm 0.06) \times 10^{-3}$ \\
1.81 & 283 & $(1.17 \pm 0.06) \times 10^{-3}$ \\
2.23 & 270 & $(1.92 \pm 0.16) \times 10^{-4}$ \\
2.23 & 295 & $(1.39 \pm 0.02) \times 10^{-3}$ \\
2.26 & 283 & $(5.13 \pm 0.28) \times 10^{-4}$ \\
2.30 & 295 & $(1.09 \pm 0.04) \times 10^{-3}$ \\
3.40 & 297 & $(9.90 \pm 0.25) \times 10^{-4}$ \\
3.91 & 296 & $(3.74 \pm 0.03) \times 10^{-4}$ \\
4.27 & 296 & $(2.99 \pm 0.02) \times 10^{-4}$ \\
5.00 & 296 & $(6.55 \pm 0.21) \times 10^{-5}$ \\
5.40 & 298 & $(2.10 \pm 0.05) \times 10^{-5}$ \\
9.26 & 313 & $(5.39 \pm 0.26) \times 10^{-7}$ \\
9.46 & 313 & $(6.07 \pm 0.28) \times 10^{-7}$ \\
10.20 & 313 & $(6.03 \pm 0.33) \times 10^{-7}$ \\
10.36 & 333 & $(4.84 \pm 0.09) \times 10^{-6}$ \\
10.37 & 325 & $(1.70 \pm 0.09) \times 10^{-6}$ \\
\hline
\end{tabular}


Author contributions. N. Borduas designed the experimental approach with critical input from J. P. D. Abbatt and J. G. Murphy on the mass spectrometry method and from J. G. Murphy and G. R. Wentworth on the ion chromatography method. N. Borduas undertook the Henry's Law experiments and B. Place conducted the HNCO hydrolysis experiments. N. Borduas analyzed the data from both sets of experiments and N. Borduas wrote the manuscript with feedback from all co-authors.

Acknowledgements. The authors acknowledge Erin Evoy for early work on the IC as well as Jim Roberts for help with interpreting Jensen's data. N. Borduas acknowledges funding from the University of Toronto Adel Sedra Graduate Fellowship. Operational support has been provided by a grant from Environment Canada. The authors also acknowledge funding from CFI and NSERC Discovery.

Edited by: A. Laskin

\section{References}

Amell, A. R.: Kinetics of the hydrolysis of cyanic acid, J. Am. Chem. Soc., 78, 6234-6238, 1956.

Barnes, I., Solignac, G., Mellouki, A., and Becker, K. H.: Aspects of the atmospheric chemistry of amides, Chem. Phys. Chem., 11, 3844-3857, 2010.

Barth, M. C., Cochran, A. K., Fiddler, M. N., Roberts, J. M., and Bililign, S.: Numerical modeling of cloud chemistry effects on isocyanic acid (HNCO), J. Geophys. Res. Atmos., 118, 86888701, 2013.

Belson, D. J. and Strachan, A. N.: Preparation and properties of isocyanic acid, Chem. Soc. Rev., 11, 41-56, doi:10.1002/jgrd.50661, 1982.

Beswick, H. T. and Harding, J. J.: Conformational changes induced in bovine lens a-crystallin by carbamylation, Biochem. J., 223, 221-227, 1984.

Borduas, N., Abbatt, J. P. D. and Murphy, J. G.: Gas phase oxidation of monoethanolamine (MEA) with $\mathrm{OH}$ radical and ozone: kinetics, products, and particles, Environ. Sci. Technol., 47, 63776383, 2013.

Borduas, N., da Silva, G., Murphy, J. G., and Abbatt, J. P. D.: Experimental and theoretical understanding of the gas phase oxidation of atmospheric amides with $\mathrm{OH}$ radicals: kinetics, products, and mechanisms, J. Phys. Chem. A, 119, 4298-4308, 2015.

Brady, J. M., Crisp, T. A., Collier, S., Kuwayama, T., Forestieri, S. D., Perraud, V., Zhang, Q., Kleeman, M. J., Cappa, C. D., and Bertram, T. H.: Real-time emission factor measurements of isocyanic acid from light duty gasoline vehicles, Environ. Sci. Technol., 48, 11405-11412, 2014.

Brownsword, R. A., Laurent, T., Vatsa, R. K., Volpp, H., and Wolfrum, J.: Photodissociation dynamics of HNCO at $248 \mathrm{~nm}$, Chem. Phys. Lett., 258, 164-170, 1996.

Dixon, R. N. and Kirby, G. H.: Ultra-violet absorption spectrum of isocyanic acid, Trans. Faraday Soc., 64, 2002-2012, 1968.

Escorcia, E. N., Sjostedt, S. J. and Abbatt, J. P. D.: Kinetics of $\mathrm{N}_{2} \mathrm{O}_{5}$ hydrolysis on secondary organic aerosol and mixed ammonium bisulfate secondary organic aerosol particles, J. Phys. Chem. A, 114, 13113-13121, 2010.

Hocking, W. H., Gerry, M. C. L. and Winnewisser, G.: The Microwave and Millimetre Wave Spectrum, Molecular Constants, Dipole Moment, and Structure of Isocyanic Acid, HNCO, Can. J. Phys., 53, 1869-1901, 1975.

Ip, H. S. S., Huang, X. H. H. and Yu, J. Z.: Effective Henry's law constants of glyoxal, glyoxylic acid, and glycolic acid, Geophys. Res. Lett., 36, L01802, doi:10.1029/2008GL036212, 2009.

Jensen, M. B.: On the kinetics of the decomposition of cyanic acid, Acta Chem. Scand., 12, 1657-1670, 1958.

Jones, L. H., Shoolery, J. N., Shulman, R. G., and Yost, D. M.: The molecular structure of isocyanic acid from microwave and infra-red absorption spectra, J. Chem. Phys., 18, 990-991, doi:10.1063/1.1747827, 1950 .

Kames, J. and Schurath, U.: Henry's law and hydrolysis-rate constants for peroxyacyl nitrates (PANs) using a homogeneous gasphase source, J. Atmos. Chem., 21, 151-164, 1995.

Kroecher, O., Elsener, M., and Koebel, M.: An ammonia and isocyanic acid measuring method for soot containing exhaust gases, Anal. Chim. Acta, 537, 393-400, 2005.

Lee, C. K. and Manning, J. M.: Kinetics of the carbamylation of the amino groups of sickle cell hemoglobin by cyanate, J. Biol. Chem., 248, 5861-5865, 1973.

Mertens, J. D., Chang, A. Y., Hanson, R. K., and Bowman, C. T.: A shock tube study of reactions of atomic oxygen with isocyanic acid, Int. J. Chem. Kinet., 24, 279-295, 1992.

Mladenović, M. and Lewerenz, M.: Equilibrium structure and energetics of CHNO isomers: steps towards ab initio rovibrational spectra of quasi-linear molecules, Chem. Phys., 343, 129-140, 2008.

Mydel, P., Wang, Z., Brisslert, M., Hellvard, A., Dahlberg, L. E., Hazen, S. L., and Bokarewa, M.: Carbamylation-dependent activation of T cells: A novel mechanism in the pathogenesis of autoimmune arthritis, J. Immunol., 184, 6882-6890, 2010.

Poppinger, D., Radom, L., and Pople, J. A.: A theoretical study of the CHNO isomers, J. Am. Chem. Soc., 99, 7806-7816, 1977.

Rabalais, J. W., McDonald, J. R., and McGlynn, S. P.: Electronic states of $\mathrm{HNCO}$, cyanate salts, and organic isocyanates. II. Absorption studies, J. Chem. Phys., 51, 5103-5111, doi:10.1063/1.1671908, 1969.

Roberts, J. M.: Measurement of the Henry's law coefficient and first order loss rate of PAN in n-octanol, Geophys. Res. Lett., 32, L08803, doi:10.1029/2004GL022327, 2005.

Roberts, J. M., Veres, P., Warneke, C., Neuman, J. A., Washenfelder, R. A., Brown, S. S., Baasandorj, M., Burkholder, J. B., Burling, I. R., Johnson, T. J., Yokelson, R. J., and de Gouw, J.: Measurement of HONO, HNCO, and other inorganic acids by negative-ion proton-transfer chemical-ionization mass spectrometry (NI-PT-CIMS): application to biomass burning emissions, Atmos. Meas. Tech., 3, 981-990, doi:10.5194/amt-3-981-2010, 2010.

Roberts, J. M., Veres, P. R., Cochran, A. K., Warneke, C., Burling, I. R., Yokelson, R. J., Lerner, B., Gilman, J. B., Kuster, W. C., Fall, R., and de Gouw, J.: Isocyanic acid in the atmosphere and its possible link to smoke-related health effects, Proc. Natl. Acad. Sci. USA, 108, 8966-8971, doi:10.1073/pnas.1103352108, 2011.

Roberts, J. M., Veres, P. R., VandenBoer, T. C., Warneke, C., Graus, M., Williams, E. J., Lefer, B., Brock, C. A., Bahreini, 
R., Öztürk, F., Middlebrook, A. M., Wagner, N. L., Dubé, W. P., and de Gouw, J. A.: New insights into atmospheric sources and sinks of isocyanic acid, $\mathrm{HNCO}$, from recent urban and regional observations, J. Geophys. Res. Atmos., 2013JD019931, doi:10.1002/2013JD019931, 2014.

Sander, R.: Modeling atmospheric chemistry: interactions between gas-phase species and liquid cloud/aerosol particles, Surv. Geophys., 20, 1-31, 1999.

Sander, R.: Compilation of Henry's law constants (version 4.0) for water as solvent, Atmos. Chem. Phys., 15, 4399-4981, doi:10.5194/acp-15-4399-2015, 2015.

Tsang, W.: Chemical kinetic data base for propellant combustion. II. Reactions involving CN, NCO, and HNCO, J. Phys. Chem. Ref. Data, 21, 753-791, doi:10.1063/1.555914, 1992.

Tully, F. P., Perry, R. A., Thorne, L. R., and Allendorf, M. D.: Free-radical oxidation of isocyanic acid, Symp. Int. Combust., 22, 1101-1106, 1989.

Veres, P., Roberts, J. M., Warneke, C., Welsh-Bon, D., Zahniser, M., Herndon, S., Fall, R., and de Gouw, J.: Development of negativeion proton-transfer chemical-ionization mass spectrometry (NIPT-CIMS) for the measurement of gas-phase organic acids in the atmosphere, Int. J. Mass Spectrom., 274, 48-55, 2008.

Veres, P., Roberts, J. M., Burling, I. R., Warneke, C., de Gouw, J., and Yokelson, R. J.: Measurements of gas-phase inorganic and organic acids from biomass fires by negative-ion proton-transfer chemical-ionization mass spectrometry, J. Geophys. Res., 115, D23302, doi:10.1029/2011JD017393, 2010.

Wang, Z., Nicholls, S. J., Rodriguez, E. R., Kummu, O., Horkko, S., Barnard, J., Reynolds, W. F., Topol, E. J., DiDonato, J. A., and Hazen, S. L.: Protein carbamylation links inflammation, smoking, uremia and atherogenesis, Nat. Med., 13, 1176-1184, 2007.
Wentzell, J. J. B., Liggio, J., Li, S., Vlasenko, A., Staebler, R., Lu, G., Poitras, M., Chan, T., and Brook, J. R.: Measurements of gas phase acids in diesel exhaust: a relevant source of HNCO?, Environ. Sci. Technol., 47, 7663-7671, 2013.

Woodward-Massey, R., Taha, Y. M., Moussa, S. G., and Osthoff, H. D.: Comparison of negative-ion proton-transfer with iodide ion chemical ionization mass spectrometry for quantification of isocyanic acid in ambient air, Atmos. Environ., 98, 693-703, 2014.

Young, P. J., Emmons, L. K., Roberts, J. M., Lamarque, J., Wiedinmyer, C., Veres, P. and VandenBoer, T. C.: Isocyanic acid in a global chemistry transport model: tropospheric distribution, budget, and identification of regions with potential health impacts, J. Geophys. Res., 117, D10308, doi:10.1029/2010JD014033, 2012.

Yu, S., Su, S., Dorenkamp, Y., Wodtke, A. M., Dai, D., Yuan, K., and Yang, X.: Competition between direct and indirect dissociation pathways in ultraviolet photodissociation of HNCO, J. Phys. Chem. A, 117, 11673-11678, 2013.

Zhao, R., Lee, A. K. Y., Wentzell, J. J. B., Mcdonald, A. M., ToomSauntry, D., Leaitch, W. R., Modini, R. L., Corrigan, A. L., Russell, L. M., Noone, K. J., Schroder, J. C., Bertram, A. K., Hawkins, L. N., Abbatt, J. P. D., and Liggio, J.: Cloud partitioning of isocyanic acid (HNCO) and evidence of secondary source of HNCO in ambient air, Geophys. Res. Lett., 41, 6962-6969, 2014. 